\title{
Optimization of Multiple Exposure Gratings for Widely Tunable Lasers
}

\author{
G. Sarlet, G. Morthier, R. Baets, Senior Member, IEEE, D. J. Robbins, and D. C. J. Reid
}

\begin{abstract}
A theoretical study of the reflection spectra of multiple holographic exposure distributed Bragg reflector gratings is presented. We will show that such a grating can exhibit a uniform reflection comb if the relative phases of the superimposed gratings are well chosen. The tolerances on the optimal phases are of the order of 0.09 radians for six superimposed gratings to 0.04 radians for ten gratings.
\end{abstract}

Index Terms - Distributed Bragg reflector lasers, holographic gratings, laser tuning, optical waveguide filters, semiconductor lasers, wavelength-division multiplexing.

\section{INTRODUCTION}

W IDELY tunable lasers are promising components for future HD-WDM networks. Most of the present widely tunable lasers employ waveguide-grating reflectors with a comb-like reflectivity spectrum [1]-[6]. Ideally, these reflectors have a number of uniform reflection peaks within a limited wavelength range. In the case of a sampled grating (SG) [1] the peak-reflectivity decreases with the distance from the central wavelength and long gratings are necessary to obtain strong reflection. On the other hand, the SG has the advantage that only a single holographic exposure is needed during processing. Super structure gratings (SSG) [2] have been designed with highly uniform reflection peaks, but these need very precise e-beam lithography for the definition of the (complex) grating pattern. Recently, the concept of the SSG has been improved by the binary superimposed gratings (BSG), which can generate similar reflection spectra, but require much less precision in the e-beam lithography [5].

In this letter, we will study the characteristics of multiple exposure Bragg gratings. These can be seen as an extension of ordinary Bragg gratings toward multiwavelength reflectors, however still relying on holographic exposure for the fabrication. A. Talneau et al. [4], [6] first presented these, but until now no theoretical calculations of reflection spectra have been presented. We will show that these multiwavelength gratings (MWG) can generate uniform reflection combs, if the relative phases of the superimposed Bragg gratings are suitably chosen. In reported experiments, e.g., [6], these phases were purely left to chance.

Manuscript received August 21, 1998; revised September 21, 1998. This work was supported in part by the ACTS-AC065 BLISS project.

G. Sarlet, G. Morthier, and R. Baets are with the Department of Information Technology, University of Gent-IMEC, B-9000 Gent, Belgium. G. Sarlet is a Research Assistant with the Fund for Scientific Research-Flanders, Belgium (F.W.O.).

D. J. Robbins and D. C. J. Reid are with GEC-Marconi Materials Technology Ltd., Caswell, Towcester, Northamptonshire NN12 8EQ, U.K.

Publisher Item Identifier S 1041-1135(99)00351-1.
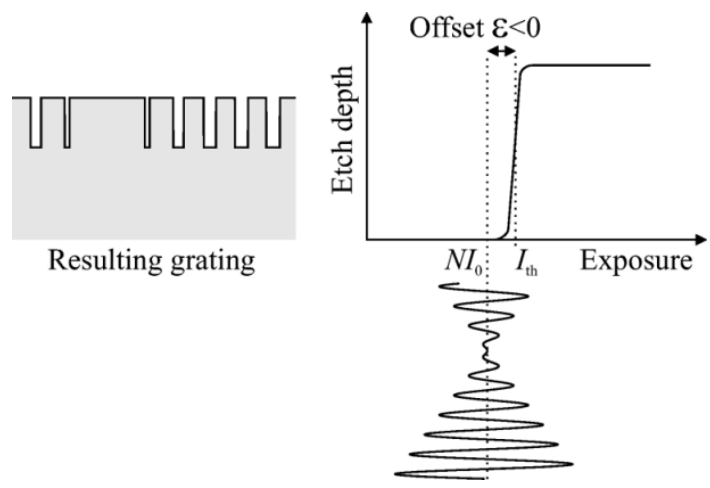

Fig. 1. Transfer of exposure intensity (integrated over time) to etch depth. Example of holographic exposure distribution for superimposed Bragg gratings and resulting grating.

\section{THEORY}

To fabricate a MWG, a thin photoresist layer is sequentially exposed by two-beam interference patterns with different pitches [4], [6]. Because of the thresholding effect of the photoresist development and the characteristics of the etching process, the transfer of the superimposed fringe patterns into semiconductor material is highly nonlinear. Especially when using dry-etching techniques, the resulting grating has a more or less rectangular profile. This means that the transfer of exposure intensity (integrated over time) to etch depth can be approximated by a step function (Fig. 1).

If the different exposure steps have equal strength (product of exposure time and average intensity is equal), the total exposure distribution as function of the position $z$ along the grating can be described by

$$
I(z)=\frac{I_{0}}{2} \sum_{k}\left\{1+\cos \left(\frac{2 \pi z}{\Lambda_{k}}+\phi_{k}\right)\right\}
$$

where $\Lambda_{k}$ is the pitch and $\phi_{k}$ is the phase of grating $k$ at a certain reference position $z=0$.

We will limit our study to those cases with an even number of superimposed gratings $2 N$ (analogous results can be found for odd numbers), with uniformly spaced frequencies centered on $F_{0}$, so that the total exposure distribution can be written as

$$
\begin{aligned}
I(z)= & N I_{0} \\
& +I_{0} \sum_{k=1}^{N}\left\{\begin{array}{c}
\cos \left(2 \pi \Delta F z\left(k-\frac{1}{2}\right)+\frac{1}{2}\left(\phi_{k}-\phi_{-k}\right)\right) \\
\cdot \cos \left(2 \pi F_{0} z+\frac{1}{2}\left(\phi_{k}+\phi_{-k}\right)\right)
\end{array}\right\} .
\end{aligned}
$$

If the gratings with frequencies $F_{k}$ and $F_{-k}$ symmetric versus the central frequency $F_{0}$ have opposite phases at $z=$ 
TABLE I

Numerical Values of Calculation Parameters

\begin{tabular}{llll}
\hline Parameter & Value & & Lnit \\
Grating center frequency $F_{0}$ & 4.2562 & $\mathrm{um}^{-1}$ \\
Grating frequency spacing $\Lambda F$ & $5.434610^{-3}$ & $\mathrm{um}^{-1}$ \\
Effective refractive index $n$ & 3.2985 & \\
Central wavelength $\lambda_{0}$ & 1.55 & $\mu \mathrm{m}$ \\
Coupling cocfficient for $2 N-6$ & $\kappa-2\left(n_{1}-n_{2}\right) / \lambda_{0}-3.00010^{-3}$ & $\mu^{-1}$ \\
Coupling cocfficient for $2 N-10$ & $\kappa=2\left(n_{1}-n_{2}\right) / \lambda_{0}=3.87110^{-3}$ & $\mu \mathrm{m}^{-1}$ \\
Weights $w_{\mathrm{k}}, 2 N-6$ (symmetrical) & $\ldots 0135101010 \ldots$ & \\
Weights $w_{\mathrm{k}}, 2 N=10$ (symmetrical) & $\ldots 0123451010101010 \ldots$ &
\end{tabular}

$0, \phi_{k}=-\phi_{-k}$, this can be simplified to

$$
I(z)=N I_{0}+I_{0} \cos \left(2 \pi F_{0} z\right) \cdot E(z)
$$

with

$$
E(z)=\sum_{k=1}^{N} \cos \left(2 \pi \Delta F z\left(k-\frac{1}{2}\right)+\phi_{k}\right) .
$$

We define the offset $\varepsilon$ as the difference between the average exposure $N I_{0}$ and the threshold $I_{\text {th }}$ (Fig. 1). In general, the result is a grating with continuously varying duty-cycle, including regions where everything is etched away $(\varepsilon>0$, overexposure) or where there is no etching $(\varepsilon<0$, underexposure).

To calculate the reflection spectra of such gratings, we used a transfer-matrix method [7]. The reflection spectrum is the same as that of a plane wave reflecting off a layered structure perpendicular to the propagation axis, with alternating layers with refractive indices $n_{1}$ and $n_{2}$, which corresponds to a grating coupling coefficient $\kappa=2\left(n_{1}-n_{2}\right) / \lambda_{0}=\pi \Delta n_{I} / \lambda_{0}$. Here, $\Delta n_{I}=2 / \pi\left(n_{1}-n_{2}\right)$ is the first order Fouriercomponent of a square wave with amplitude $n_{1}-n_{2}$. The calculation of the reflection spectrum generally involves the multiplication (for each wavelength) of several thousands of transfer-matrices, as the alternating layers have a continuously varying thickness. In the special case with anti-symmetric phases $\phi_{k}=-\phi_{-k}$ and offset $\varepsilon=0$ however, nearly all layers have the same thickness and therefore the long sequence of transfer matrices can be regrouped analytically into a sequence of only a few tens of matrices, which greatly reduces computation time.

\section{CAlculation Examples}

The simplest example of a multiwavelength grating is the Moire grating, where $2 N=2$. Fig. 2(a) shows the reflection spectrum. ${ }^{1}$ For $2 N>2$ the relative phases $\phi_{k}$ begin to play a role. Compare for example the Fig. 2(b), (c), and (d) where $2 N=6$. In Fig. 2(b) all phases are 0, while in Fig. 2(c) we have made $\phi_{3}=-\phi_{-3}=\pi$. Fig. 2(d) shows the reflection spectrum for a set of arbitrarily chosen phases. If the aim is to obtain $2 N$ equally strong reflection peaks at the wavelengths corresponding to the different holographic pitches $\Lambda_{k}$, the spectrum of Fig. 2(c) is clearly much better than that of Fig. 2(b) and (d).

\footnotetext{
${ }^{1}$ Numerical values used for different calculation parameters are given in Tables I and II
}

TABLE II

Optimized Phases for Several Values of the Number of Peaks $2 N$

\begin{tabular}{c|cccccc}
\hline $2 N$ & $\phi_{10}$ & $\phi_{20}$ & $\phi_{30}$ & $\phi_{40}$ & $\phi_{50}$ & $\phi_{60}$ \\
\hline 2 & 0 & & & & & \\
4 & 0 & -1.844 & & & & \\
6 & 0 & -0.350 & -2.542 & & & \\
8 & 0 & -2.646 & -0.981 & -2.943 & & \\
10 & 0 & -1.713 & -0.148 & -0.069 & +3.100 & \\
12 & 0 & -1.351 & 2.276 & -0.943 & +0.974 & +1.169 \\
\hline
\end{tabular}

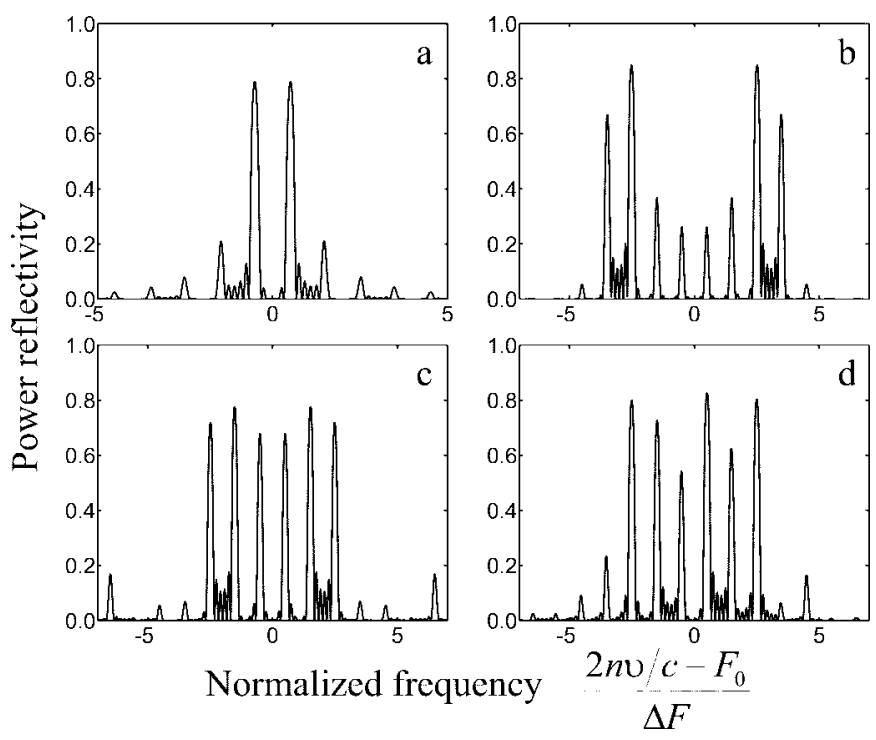

Fig. 2. Examples of reflection spectra of 2 (a) or $6(\mathrm{~b}, \mathrm{c}, \mathrm{d})$ superimposed Bragg gratings. (a) $\left[\phi_{1} \phi_{-1}\right]=[00]$, (b) $\left[\phi_{3} \cdots \phi_{-3}\right]=\left[\begin{array}{llllll}0 & 0 & 0 & 0 & 0 & 0\end{array}\right]$, (c)

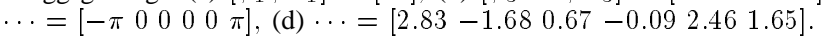

Another important parameter is the grating length $L$. The absolute value of the envelope function $E(z)$ is periodic with a (super)period $1 / \Delta F$. For the reflection peaks to be sufficiently narrow and for the spectrum to be more or less independent of the position of the grating edge within the superperiod, the total length $L$ should be at least a few (e.g., 5) times this period. In our calculations we took $L=6 / \Delta F$.

\section{OPTIMIZATION}

Here, we will discuss the optimization of the reflection spectrum as function of the phases $\phi_{k}$, where the objective is to obtain equally strong reflection peaks at the wavelengths corresponding to the different holographic pitches $\Lambda_{k}$, and weak reflection at other wavelengths. The optimization was done for gratings with antisymmetric phases $\phi_{k}=-\phi_{-k}$ and offset $\varepsilon=0$, because of the reduced computation time and more importantly because only these gratings have symmetric reflection spectra. We made $\phi_{1}=-\phi_{-1}=0$, as adding a value of $(2 k-1) \theta$ to $\phi_{k}(k \geq 1, \theta$ any arbitrary angle) and maintaining the antisymmetry, would give the same grating, only shifted over a distance $\Delta z=\theta /(\pi \Delta F)$.

As cost function we took a weighted sum of squared errors between the peak reflectivities $R_{ \pm k}$ at the frequencies

$$
v_{ \pm k}=\frac{c}{2 n}\left[F_{0} \pm\left(k-\frac{1}{2}\right) \Delta F\right]
$$




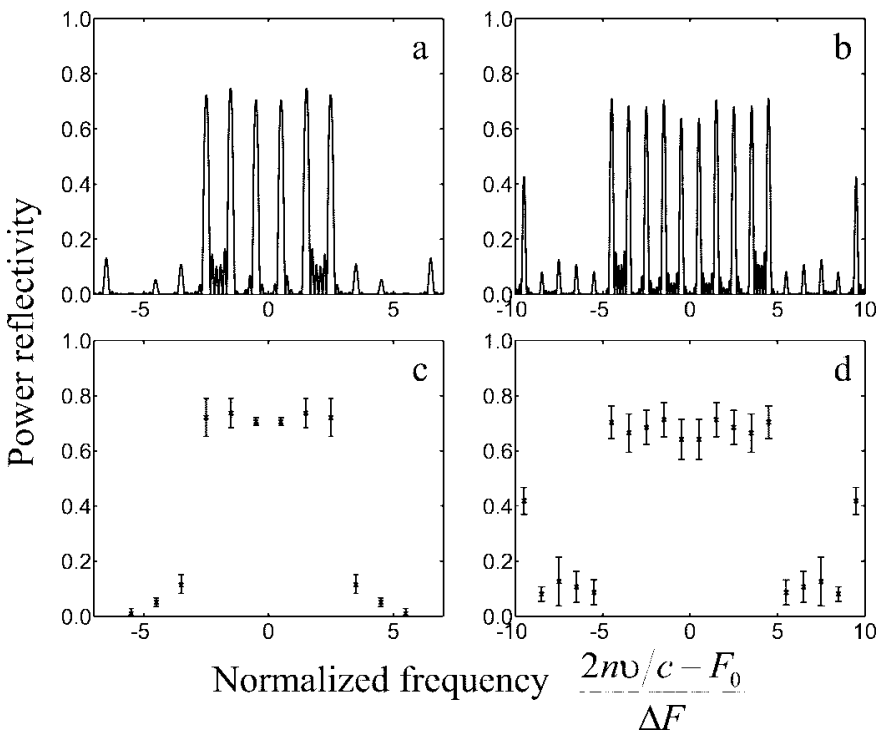

Fig. 3. Optimized reflection spectra for (a) 6 and (b) 10 superimposed Bragg gratings. Error bars (95\% probability intervals) for Gaussian errors on the relative phases with a standard deviation (c) $\sigma=2 \pi / 100$ resp. (d) $\sigma=2 \pi / 200$.

and $R_{\max } \delta_{ \pm k}$, where $R_{\max }$ is the overall maximum reflectivity, $\delta_{ \pm k}=1$ for $1 \leq k \leq N$ and $\delta_{ \pm k}=0$ for all other $k$

$\operatorname{cost}=\sum_{k}\left\{w_{k}\left(R_{\max } \delta_{k}-R_{k}\right)^{2}+w_{-k}\left(R_{\max } \delta_{-k}-R_{-k}\right)^{2}\right\}$.

We minimized this function using the Nelder-Mead simplex search algorithm [8], with randomized starting values for $\phi_{2}, \cdots, \phi_{N}$ to make sure that we find a global minimum.

Fig. 3(a) and (b) show the optimized reflection spectra for $2 N=6$ and $2 N=10$. In order to estimate the tolerances on the optimum phases $\phi_{k: 0}$, we calculated multiple reflection spectra when Gaussian errors with a given standard deviation $\sigma$ were added to the optimum phases. Fig. 3(c) and (d) show the intervals that contain $95 \%$ of the calculated peak reflectivities for the standard deviations $\sigma=2 \pi / 100$ for $2 N=6$ and $\sigma=2 \pi / 200$ for $2 N=10$. In both cases the maximum error is approximately $10 \%$ of the maximum reflectivity $R_{\max }$. As could be expected, the tolerances on the phases $\phi_{k 0}$ become tighter as the number of peaks $2 N$ increases: the tolerances for a maximum reflectivity deviation of $0.1 R_{\max }$ are of the order of 0.09 radians for six peaks, decreasing to 0.04 radians for ten peaks (a 95\% probability corresponds to a maximum phase deviation of approximately $1.4 \sigma$ ).

Finally, we investigated the influence of a nonzero offset $\varepsilon$ on the reflection. It appears that the peak-reflectivities decrease, which is caused by the decrease of the effective coupling coefficient for an average grating duty-cycle different from 50\%. Also, the reflectivities for the longer (shorter) wavelengths are slightly stronger for positive (negative) $\varepsilon$, and the parasitic reflection peaks are strongly suppressed.

\section{CONCLUSION}

The reflection spectra of multiwavelength gratings (MWG's) have been studied theoretically. Some general design rules were derived: the grating length $L$ should be at least a few times the "superperiod" $1 / \Delta F$, and the relative phases $\phi_{k}$ of the superimposed Bragg gratings should be carefully controlled. Numerical optimization of the relative phases $\phi_{k}$ resulted in uniform reflection combs with peaks at all the corresponding Bragg wavelengths. Tolerances on the optimized phases $\phi_{k: 0}$ are of the order of 0.09 radians for six peaks to 0.04 radians for 10 peaks.

\section{REFERENCES}

[1] V. Jayaraman, Z. M. Chuang, and L. A. Coldren, "Theory, design and performance of extended tuning range semiconductor lasers with sampled gratings," IEEE J. Quantum Electron., vol. 29, pp. 1824-1834, June 1993.

[2] H. Ishii, H. Tanobe, F. Kano, Y. Tohmori, Y. Kondo, and Y. Yoshikuni, "Quasicontinuous wavelength tuning in super-structure-grating (SSG) DBR lasers," IEEE J. Quantum Electron., vol. 32, pp. 433-440, Mar. 1996.

[3] M. Öberg, S. Nilsson, K. Streubel, L. Bäckbom, and T. Klinga, "74 nm wavelength tuning range of an $\mathrm{InGaAsP} / \mathrm{InP}$ vertical grating assisted codirectional coupler laser with rear sampled grating reflector," IEEE Photon. Technol. Lett., vol. 5, pp. 735-738, July 1993.

[4] A. Talneau, C. Ougier, and S. Slempkes, "Multiwavelength grating reflectors for widely tunable laser," IEEE Photon. Technol. Lett., vol. 8, pp. 497-499, Apr. 1996.

[5] I. A. Avrutsky, D. S. Ellis, A. Tager, H. Anis, and J. M. Xu, "Design of widely tunable semiconductor lasers and the concept of binary superimposed gratings (BSG's)," IEEE J. Quantum Electron., vol. 34, pp. 729-741, Apr. 1998

[6] A. Talneau, J. Charil, and A. Ougazzaden, "Superimposed Bragg gratings on semiconductor material," Electron. Lett., vol. 32, no. 20, pp. 1884-1885, 1996.

[7] T. Makino, "Transfer-matrix formulation of spontaneous emission noise of DFB semiconductor lasers," J. Lightwave Technol., vol. 9, pp. 84-91, Jan. 1991.

[8] J. A. Nelder and R. Mead, "A simplex method for function minimization," Comput. J., vol. 7, pp. 308-313, 1964. 